\title{
From the Visual Book to the WEB Book: the Importance of Good Design
}

\author{
M. Landoni ${ }^{*}$, R. Wilson ${ }^{+}$and F. Gibb. \\ * Department of Information Science, University of Strathclyde. \\ ${ }^{+}$CDLR: Centre for Digital Library Research, University of Strathclyde.
}

\begin{abstract}
This paper presents the results of two studies into electronic book production. The Visual Book study [1] explored the importance of the visual component of the book metaphor for the production of more effective electronic books, while the WEB Book study [2] took the findings of the Visual Book and applied them to the production of books for publication on the WWW. Both studies started from an assessment of which kinds of paper book are more suitable for conversion into electronic form, and both identified as target publications those which are meant to be used for reference rather than those which are read sequentially and usually in their entirety by users. This includes scientific publications and textbooks which have been chosen for the Visual Book and the WEB Book experiments. In this paper we discuss the results of the two studies and the way they could influence the design and production of more effective electronic books.
\end{abstract}

\section{Introduction}

The result of integrating the classical book structure with features which can be provided within an electronic environment constitutes a generally accepted definition of an electronic book. The main features of electronic books are that they are dynamic, reactive, can be made available in different formats and/or editions in a short time and, with the growth of the Internet, are accessible almost everywhere [3],[4]. However the cognitive overhead associated with a computer-based environment is an important reason for carefully considering the appropriateness and the method of realising this conversion [5],[6]. It is important to consider both the subject matter and the intended usage of a particular paper book in order to decide whether an electronic version will be useful or not [7], [8]. It seems clear that the desire or willingness to use electronic books is primarily based on the need for rapid access to information chunks rather than for assimilating significant amounts of narrative text [9]. An analysis of the market for paper and electronic publications showed that users prefer to keep a paper version in addition to the electronic one even when a free electronic version is available on WWW [10].

The motivations for producing electronic books therefore deal with factors related to the market requirement for electronic books, as well as cognitive issues related to the ability of the reader to use, appreciate and prefer books in electronic format to papers ones [11]. The quality aspect becomes crucial and for this reason the design process 
has to be reviewed in order to create effective electronic books, i.e. communicators of electronic information which have additional value which paper cannot provide. This paper describes two studies into the design and production of electronic books: the Visual Book and the WEB Book. The next three sections discuss the theory behind the Visual Book experiment, its design and findings: this provides the background for the description of the WEB Book experiment. Finally conclusions are drawn from both experiences.

\section{Visual Rhetoric}

The Visual Book experiment was a study into the application of the book metaphor to the design and production of electronic books. The novel aspects of the Visual Book studies was the importance given to the visual components of the physical book when designing electronic book. The idea behind visual rhetoric [14] is to use visual clues to identify or highlight those parts of a document which are more important for the comprehension of its meaning. Previous research has corroborated this view that a document can be interpreted as a visible representation of a text according to its semantic contents [15]. Visual rhetoric provides the reader with a graphical mark up language which is immediately recognisable on the basis of previous reading activity. Different graphical presentations suggest different readings and deeply affect the interpretation of the contents of the same text. The Visual Book project focused on the importance and use of visual rhetoric when presenting information on a screen and in particular on the influence of visual rhetoric in the presentation and use of electronic books.

\section{The Visual Book Experiment}

The Visual Book represents a particular interpretation of the electronic book, based mainly on the visual aspects of the paper book such as dimensions, thickness, page form and general design style [1]. A Visual Book is the result of the process of converting an existing paper book into electronic form using two main components:

- The Visual Book Builder: the authoring system for building the Visual Book.

- The Visual Book Browser: the module for presenting the Visual Book to readers.

The physical aspect of the paper book is the key to the construction and representation of a Visual Book. Emphasis is placed on preserving the book's appearance in order to draw upon the familiarity the reader already has with books in general. Book functionalities were studied in terms of how books are used in practice to ensure that information is presented in a natural and familiar way and that the reading process is supported in an amenable environment.

The main idea of a Visual Book is to offer the reader an object as similar as possible to the paper book by replacing its essential physical features, such as size and quality, with visual ones [16]. Particular attention was paid to identifying those functionalities a Visual Book could acquire from electronic support which would be most useful and consistent with the specification of the book concept. One of the main advantages of 
electronic support is the ease with which the designer can modify, update and distribute data [17]. On the other hand there is a series of functionalities, which already form part of the paper book environment, that are supported only in part by other electronic book models. These include:

- Bookmarks.

- Notes on the margins or elsewhere in the text.

- Highlighting of interesting parts.

- Easy access to pages which are frequently consulted by following different visual clues.

- Information about the ratio between the pages already read and those remaining.

- Control of the reading progress.

- Browsing and scanning for interesting sections.

All of these have been incorporated as essential features of the Visual Book Browser, as shown in Figure 1. In particular search tools, such as an Index tool and Table of Contents (ToC), have been added to the Visual Book features.

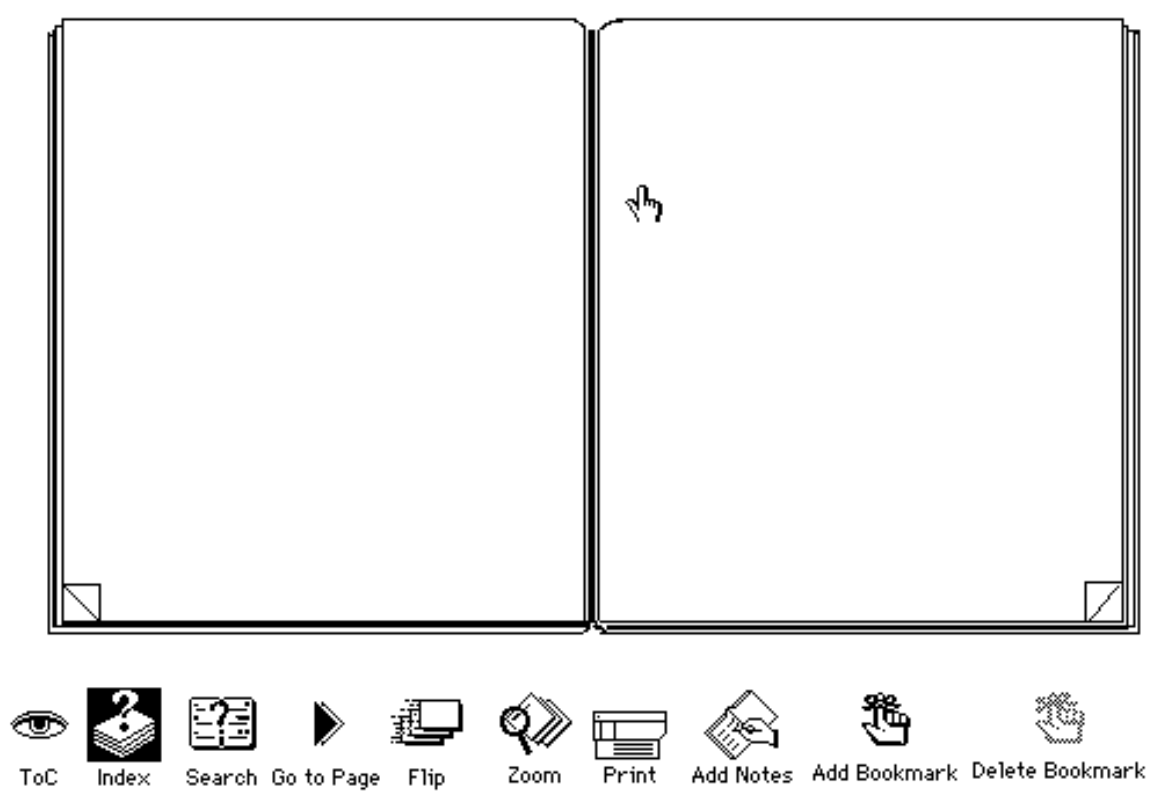

Fig. 1. The Visual Book Browser

\section{Visual Book Findings and Analysis}

The evaluation of the Visual Book [1] concentrated on aspects of the book metaphor when converting paper books into electronic format. In particular the importance of maintaining the same typographical features, the design rules, the pagination format and the paper appearance were considered carefully in terms of retaining the meaning of the original text. The physical aspects of the page are encapsulated in a set of 
presentation rules through which visual clues give the reader semantic information about the context. This primary feature of the book metaphor has been considered to be particularly important and to be an original contribution to the future development of the electronic design process.

A number of criteria were extracted from other evaluations of the processes used when reading from a computer [18], [19] and these were used as the basis for the Visual Book evaluation:

- Sense of directness is the degree of feeling users have that changes on the screen are the result of their actions. It is connected with the illusion users have that the displayed image is a physical object which can be manipulated in the same way as a real paper book.

- Sense of engagement is the level of interest the system induces in users. One source of engagement is the fun of seeing the system react and is related to the novelty of the system; tangibility and responsiveness are also responsible for a good level of engagement. Paper has generally a low level of engagement because it is not interactive and is overly familiar to users.

- Sense of text is the feeling users may have of the structural and semantic structure of the text that is being read, i.e. its spatial disposition. Readers are known to be able to recall the position of text in a paper text [20]. Factors which can influence the sense of text are the page size, legibility, and a low responsiveness while scrolling when looking for more text.

In general the results of the evaluation of the Visual Book, which were supported by the findings of a similar project - the Hyper-Book [21], showed that the book metaphor was both accepted and understood by its evaluators. These results also highlighted the necessity for a new role in electronic publishing, "the designer of electronic books, as the person in charge of the final appearance of the electronic book". This is a person competent not only in the technological aspects of producing electronic documentation but with a background in understanding the importance of presentation issues such as pagination and the general format and appearance of the electronic document, which can be called collectively electronic typography. This means that typographical rules are essential components of the cognitive model of a book and hence relevant to the conversion of the metaphor of the book into electronic form.

The evaluation method which was applied to the Visual Book was the cognitive jogthrough [22]. In this approach, users were free to express, in written or verbal form, their comments and suggestions. Users were happy to interact with an object which resembled a book and appreciated that its enhanced functionalities were consistent with the original paper version. The main request was for an intelligent search function to combine the precision provided by paper visual clues and by index terms with the high recall provided by full text search which can be performed very efficiently using mainstream information retrieval software.

In addition to the comments from evaluators a number of future developments were identified by the research team based on their experiences. In particular the influence of the Internet on the presentation of information in electronic format should be studied in order to understand if modifications to the book metaphor and the related concept of visual rhetoric have to be considered to make it compatible with the 
Internet paradigm. It is this issue that the WEB Book investigated and which is described in the next section.

\section{The WEB Book Experiment}

The WEB Book was based on the central hypothesis of the Visual Book project: that appearance is an important factor in the effective presentation of information on screen, and applied it to the production of books on the WWW. The aim was to study whether focusing on the appearance of the content when preparing a scientific textbook for electronic publication has a positive impact on its usability.

A series of studies by Morkes and Nielsen [12],[13] into how to write for the Web provided the basis for a methodology which would enable any improvement to be measured. They proposed that the usability of a text on the Web can be greatly increased by altering the presentation of the information to make it concise, scannable and objective. Their definition of what concise, scannable and objective text is as follows:

- Concise text is where less-important information is edited out to reduce the page length.

- Scannable text is written to encourage scanning, or skimming, of the text for information of interest. For this purpose bulleted lists, boldface text to highlight keywords, photo captions, shorter sections of text, and more headings, etc. are used.

- Objective text presents information without exaggeration, or making subjective or unsustainable claims.

When applied in their own experiments to a site with tourist information and a site with technical white papers (i.e. papers in progress), these changes led to increases in usability of $124 \%$ and $159 \%$ respectively. The WEB Book project adhered to their procedure, as described in Applying Writing Guidelines for Web Pages [13] as closely as possible, and replicated the content of the questionnaires from their third study, using Chapter Five (Search Strategies) of van Rijsbergen's Information Retrieval [23] as the material for the experiment ${ }^{1}$. This textbook is one of the fundamental readings of IR, and a relatively plain machine readable version of the book already exists on the Internet.

Some modifications to Morkes and Nielsen's original procedures were necessary to suit the purposes of this research ${ }^{2}$. Because a scientific textbook is primarily objective in nature, and cannot be made concise without either breaching copyright or compromising the meaning of the text, the emphasis in the WEB Book was simply on reworking the chapter to make it scannable by improving/redesigning its logical and physical layout. As the experiment was being conducted on a different type of material, its objectives varied from those of the original studies, so that browsing rather than searching for information in the text was encouraged, making

\footnotetext{
${ }^{1}$ Permission to use this material by the author is gratefully acknowledged.

2 Revised version of the questionnaires used can be found at: http://www.dis.strath.ac.uk/students/ruth/forma.html.
} 
measurement of the time taken to complete the tasks less central to the purpose of the study.

The resulting methodology was used to test three hypotheses:

- Hypothesis 1: Users of the revised, scannable version of the chapter will make fewer errors on tasks than will users of the original version.

- Hypothesis 2: Users of the revised version will report higher subjective satisfaction with the site than will users of the original.

- Hypothesis 3: When measures from the first two hypotheses are combined into an overall usability score for each version of the site, the scannable version will have a higher usability score than the control version (i. e. the original electronic version).

Each stage of this experiment was carried out over the Internet. Participants consisted of 18 respondents to emails sent to two mailing lists for the IR community and to a group of postgraduate students who had studied IR in the Department of Information Science, University of Strathclyde. By focusing on the IR community the intention was to target users familiar with the general subject matter of the textbook they would be asked to read, so that they could concentrate on browsing the site for information rather than being distracted by entirely alien content. Although mailing list respondents inevitably comprised a greater number of experts, similar numbers in each group had consulted van Rijsbergen's book before and the students were considered to have sufficient background in the field to be valid subjects in the experiment.

Two electronic versions of Chapter Five were used in this study: the original ${ }^{3}$, and a revised version, written for the purpose of the experiment ${ }^{4}$. Of the eighteen participants in the experiment eight looked at the original version of Information Retrieval, and ten looked at the revised version.

\section{Design Issues for the WEB Book}

In the original electronic version only minor concessions had been made to the new medium. Each chapter is allocated a separate "page" (i.e. a web page) and text is organised in a linear way so that users have to keep scrolling down until they reach the end of the chapter. Hypertext functions provided by Web browsers are used to provide certain basic structural links, such as moving from one chapter to the next. There are links from citations to bibliographic references, and links from the table of contents to the beginning of chapters have been inserted. Otherwise, it does not provide any more effective functionalities than those provided in the paper book.

The revised version of this chapter was prepared for this study by the research team and followed Morkes and Nielsen's guidelines for improved scannability. The chapter was divided into ten separate files (Introduction, Boolean search, and so on) according to the sub-headings in the original text, so that each sub-section occupied a separate page. In addition, a chapter contents page was created to list and provide

\footnotetext{
${ }^{3}$ Available at: URL http://www.dcs.gla.ac.uk/Keith/Chapter.5/Ch.5.html.

${ }^{4}$ Available at: URL http://www.dis.strath.ac.uk/students/ruth/chpt5/.
} 
links to each section. Hyperlinks were exploited in order to aid navigation. At the top of each page, links were inserted to enable the user to jump back to the previous section, forward to the next section, or back to the table of contents for the chapter. At the foot of each page there was a list containing hyperlinks to each section in the chapter. Extra headings, coloured and sized, were inserted in the text in order to divide it into smaller, more digestible chunks. All diagrams were centred to give the pages a neater, more symmetrical appearance, and in order that they could be easily distinguished from the text. Key words and phrases were coloured red to call attention to important pieces of text, and lists were indented, their numbers or bullets also coloured red. These changes were intended to make the chapter more easily scannable by giving it a design which exploited more fully the functions of the Web as a medium.

\section{The WEB Book: Findings and Analysis}

The two major measures used to calculate the overall usability of each version of the chapter were the percentage of errors made in the search tasks, and subjective satisfaction (the culmination of all the individual elements of the questionnaire). These results are presented in Table 1.

Table 1. Mean scores for the two major measures, with standard deviations in parentheses. (Task errors are percentage scores, and subjective satisfaction are the mean scores from the questionnaires, on a scale from one to 10).

\begin{tabular}{|l|l|l|}
\hline Condition & Task Errors & Subjective Satisfaction \\
\hline Original & $16.7(17.8)$ & $5.1(1.4)$ \\
\hline Revised & $6.7(14.0)$ & $7.6(0.8)$ \\
\hline
\end{tabular}

To determine how much better or worse in percentage terms the revised chapter was relative to the control, Morkes and Nielsen's procedure for normalising the participant groups' mean scores for the two measures was adopted. For each measure, the revised condition's mean scores were transformed by division relative to the control (see Table 2) so that scores above 100 are better than the control, and those below 100 are worse.

Again following the original experiment, the overall usability score for each version was calculated by taking the geometric mean of the normalised scores for the two measures. (The geometric, rather than arithmetic, mean was used because ratios were being compared). Table 2 shows that $150 \%$ fewer task errors were made by users of the revised version, and these participants recorded $48 \%$ higher subjective satisfaction. Combined, this means an increase in overall usability of $92 \%$. The chapter which was rewritten to take into account the appearance of the content so that it was more scannable, achieved a higher score in every single measure than the original version of the text, and the three hypotheses were substantiated:

- Hypothesis 1 was verified. Users of the revised version made 150\% fewer errors on tasks than users of the original version. 
- Hypothesis 2 was supported. Users of the revised version reported 48\% higher subjective satisfaction than users of the original version.

- Hypothesis 3 was confirmed. Overall usability scores for the two versions of the site show that, compared to the control version, the revised version was $92 \%$ better.

Table 2. Normalised mean scores for the two major measures and overall usability.

\begin{tabular}{|c|c|c|c|}
\hline Condition & Task Success & Subjective Satisfaction & Overall Usability \\
\hline Original & 100 & 100 & 100 \\
\hline Revised & 250 & 148 & 192 \\
\hline
\end{tabular}

Participants' comments were in accordance with these results. Users of the original version of the chapter disliked the design of the pages, describing it variously as "flat and not very appealing", "very user-unfriendly" and "plain", with formulae that are difficult to read, making it hard to concentrate on searching for information. Users of the revised version, on the other hand, made some positive remarks about the page design, describing it as "easy to navigate" and "clean-looking". Problems with the site were also identified, with one participant commenting on the problem of having to scroll up and down to compare the different diagrams and equations.

In this case study, making the chapter more scannable according to Morkes and Nielsen's guidelines led to a $92 \%$ increase in usability, indicating the positive impact of focusing on the appearance of the content when preparing a scientific textbook for publication on the Web. Although the experiment was conducted on a small scale, these results are indications of the potential effect on usability of altering the appearance of texts on the Web to make them more scannable. It would benefit from being replicated on a larger scale, perhaps on the whole textbook, and with users from a wider range of backgrounds. Nonetheless, these indications are positive and in tune with the findings of previous exercises which form the background to the experiment. They reinforce the hypothesis of Crestani and Melucci's Case Study of Automatic Authoring [24] in which it is suggested that textbooks, because they are often used for accessing specific facts and pieces of information, are more effectively represented electronically in a non-linear fashion. Further, this research is in accordance with a key premise of the Visual Book project, which states that a book's appearance is a main factor in the specification of interfaces designed to present information which has been published on paper.

\section{Conclusions}

The aim of the two projects described in this paper is to promote the definition of guidelines for the production of more effective electronic books. The definition of

5 This was "Task Error" in Morkes and Nielsen original study, but it was changed to avoid misunderstanding. 
what an electronic book is, or has to be, is quite loose and unfortunately this is unavoidable because of the nature of the object and the difficulty of defining something with so many aspects and functionalities. In general the perspective of the whole Visual Book and WEB Book projects is centred on readers and their needs, expectations, cognitive models and requirements. The results of both experiments have shown that the book metaphor plays a crucial role in the design of electronic books.

In particular:

- The page metaphor should be respected. That is, the page should be treated as a visual space where information can be easily found and scanned.

- The logical structure of the book has to be considered. Table of Contents and Index(es) are essential features. These cannot be simply replaced by search facilities whose complexity makes the reading process more confusing for the user.

- The book template has to be used strictly to present information which is book related, and not any other kind of material as the result would be a heterogeneous system which could confuse users.

- Titles, pagination, typographical aspects have to be designed carefully to enhance text readability and scannability.

- Visual clues have to be adapted to exploit the potential of the medium where the book is published.

These observations were originally derived from the Visual Book experience but have proven to be flexible enough to be applied to the WEB Book. In fact they could be summarised in what Morkes and Nielsel [12],[13] recognised as the need for scannable text when the electronic book is going to be delivered on the WWW. Presentation and appearance play a crucial role in allowing users to read and use text in electronic form as much as they did and do with information on paper. Thus it is not surprising that guidelines for the production of good electronic books will have to take into account issues related to style and layout. The authors of this paper believe that greater emphasis on the observation of readers' reactions, can lead to the definition of heuristic rules for the production of "good" and "readable" electronic books.

\section{References}

1.Landoni, M.: The Visual Book system: A study of the use of visual rhetoric in the design of electronic books. Glasgow: Department of Information Science of the University of Strathclyde (PhD Thesis). (1997).

2.Wilson, R.: The importance of appearance in the design of WEB books. Glasgow: Department of Information Science of the University of Strathclyde (MSc Diss.) (1999).

3.Barker, P.: Interactive Electronic Books. Interactive Multimedia, 2(1) (1991) 11-28.

4.Barker, P.: Living books and dynamic electronic libraries. The Electronic Library, 14(6) (1996) 491-501.

5.Barker, P., Richardson, S. and Benest, I.D.: Human-computer interface design for electronic books. In: Raitt, D.I. and Jeapes, B. (eds.). Proceedings of the Online Information 94, 16th International Online Information Meeting, London 6-8 December. Oxford: Learned Information (1994) 213-292. 
6.Benest, I.D., Morgan G., and Smithurst M.D.: A humanised interface to an electronic library. In: Bullinger, H. J. and Shackel, B. (eds.): Proceedings of INTERACT'87. Amsterdam: Elsevier Science (1987) 905-910.

7.Wilson, T.: Electronic publishing and the future of the book. Presented at the International Conference on Book Science, Vilnius University, Lithuania. (1997). Available at: http://www.shef.ac.uk/ is/publications/infres/paper39.html (Last visited 1/7/99).

8.Landoni M., Crestani F. and Melucci M.: The Visual Book and the Hyper-TextBook: Two Electronic Books One Lesson?. In: Proceedings of RIAO'2000 Conference on Contentbased Multimedia Information Access, Paris, April 12-14. Paris: C.I.D. (2000) 247-265.

9.Leaver, R.: The future of electronic publishing for book publishers within Britain. Aslib proceedings. 47 (7/8). July/August. (1995) 164.

10. Pope, B. K.: How to succeed in online market- National Academy Press: A Case Study. The Journal of Electronic Publishing. June. 4(4) (1999). Available at: URL http://www.press.umich.edu/jep/04-04/pope.html.

11. Catenazzi, N., Landoni, M. and Gibb, F.: Design issues in the production of hyper-books and visual-books. Alt-J Association for Learning Technology Journal 1(2) (1993) 40-54.

12. Morkes, J. and Nielsen, J.: Concise, SCANNABLE, and objective: how to write for the Web. Available: URL http://www.useit.com/papers/webwriting/writing.html (Last visited 11/6/99) (1997).

13. Morkes, J. and Nielsen, J.: Applying writing guidelines to Web pages. Available: URL http://www.useit.com/papers/webwriting/rewriting.html (Last visited 11/6/99) (1998).

14. Landoni M. and Gibb F.: The Importance of Visual Rhetoric in the Design and Production of Electronic Books: The Visual Book Experience. The Electronic Library. In press. (2000).

15. Southall, R.: Interfaces between the designer and the document. In: André, J., Furuta, R. and Quint, V. (eds.). Structured documents. Cambridge: Cambridge University Press (1989) 119-131.

16. Landoni, M., Catenazzi, N. and Gibb, F.: Hyper-books and visual-books in an electronic library. The Electronic Library, 11(3) (1993) 175-186.

17. Catenazzi, N. and Gibb F.: The publishing process: the hyper-book approach. Journal on Information Science. 21(3) (1995) 161-172.

18. Hansen, W.J. and Haas, C.: Reading and writing with computers: a framework for explaining differences in performance. Communication of ACM, 37(9) (1988) 1080-1089.

19. Egan, D.E., Lesk, M. E., Ketchum, R. D., Lochbaum, C. C., Remde, J. R., Littman, M., and Landauer T. K.: Hypertext for the Electronic Library? CORE Sample Results. In: Proceedings of Hypertext '91, San Antonio. New York: ACM Press (1991) 299-312.

20. Rothkopf, E. Z.: Incidental Memory for location of information in text. Journal of Verbal Learning and Verbal Behaviour, 10(6), (1971) 608-613.

21. Catenazzi, N.: A Study into electronic book design and production: Hyper-Book and HyperBook Builder. Glasgow: Department of Information Science of the University of Strathclyde (PhD Thesis) (1994).

22. Rowley, D. E. and Rhoades, D. G.: The cognitive jog-through: a fast-paced user interface evaluation procedure. In: Proceedings of CHI'92 Conference on Human Factors in Computing Systems, Monterey, May 3-7. New York: ACM Press (1992) 389-395.

23. van Rijsbergen, C.J.: Information retrieval. 2nd Edition, London: Butterworths (1979). Available at: URL http://www.dcs.gla.ac.uk/Keith. (Last visited 30/9/99).

24. Crestani, F. and Melucci, M.: A case study of automatic authoring: from a textbook to a hyper-textbook. Data and Knowledge Engineering. 27(1) (1998) 1-30. 